\title{
Chloride Intracellular Channel Protein 1 and its Role in Neurodegenerative Disorders and Cancerous Tumors
}

\author{
Hillary Nguyen ${ }^{1,3}$, Antonio Lauto ${ }^{2,3}$, Anu Shanu ${ }^{1,4}$ and Simon J Myers ${ }^{1,3,4 *}$ \\ ${ }^{1}$ Neuro-Cell Biology Laboratory, Western Sydney University, Australia \\ ${ }^{2}$ Biomedical Engineering and Neuroscience (BENS), Western Sydney University, Australia \\ ${ }^{3}$ School of Science and Health, Western Sydney University, Australia \\ ${ }^{4}$ Molecular Medicine Research Group, School of Medicine, Western Sydney University, Australia
}

\begin{abstract}
Chloride intracellular channel protein 1 (CLIC1) is a highly conserved intracellular anion channel protein, thought to perform significant roles in maintaining cellular homeostasis. The novelty of its properties by which it can exist in soluble globular form and as integral membrane protein have earned it much interest. While the absolute functional role of CLIC1 is still debated, it is undoubtedly established that activation of CLIC1 increases membrane chloride ion permeability. The versatility of its redox regulated structural transitions has led to its addition to the rare category of metamorphic proteins. Although the exact functions of CLIC1 in maintaining cellular homeostasis still remain to be elucidated, several studies strongly indicate the possible involvement of CLIC1 up regulation in various disease states including cancer and neurodegenerative disorders, implying its significance as a potent drug target. The objective of this review is to explore its structural novelty, regulation and roles in pathologies delineating its significance in neurodegenerative diseases.
\end{abstract}

Keywords: Chloride intracellular channel protein 1; Chloride efflux; Reactive oxygen species; Cancerous tumor; Microglial neurotoxicity; Neurodegeneration

Abbreviations: CLIC1: Chloride Intracellular Channel Protein-1; GSH: Glutathione; GST: Glutathione-S-Transferase; Pro: Proline; Gln: Glutamine; Cys: Cysteine; IAA: Inadanyloxyacetic Acid; ER: Endoplasmic Reticulum; ROS: Reactive Oxygen Species; ERK: Extracellular Signal Related Kinase; A $\beta$ : Beta Amyloid; ALS: Amyotrophic Lateral Sclerosis

\section{Introduction}

Chloride intracellular channel protein 1 (CLIC1) also called nuclear chloride ion channel 27 (NCC27), is a protein encoded by the CLIC1 gene exhibiting chloride ion channel activity and is included under a broader class of chloride intracellular channels (CLICs) [1]. Under normal reducing conditions, CLIC1 it is found in a soluble, globular form in cytoplasm whereas under oxidizing conditions it is subjected to structural reconfiguration undergoing membrane insertion within the plasma membrane and other internal membranes, including the nuclear membrane [2]. CLIC1 predominantly localizes in the nucleoplasm, nuclear membrane, cell membrane and cytoplasm [3]. Nevertheless, the protein can also be found within small intra-cytoplasmic organelles, including lysosomes, endosomes and secretory vesicles [1].

Despite little being known about the biology of the CLIC proteins, its high degree of conservation across a wide range of species is indicative of its biological significance [4], by which it continues to perform essential functions in humans and other mammalian species [5]. These functions include secretion and absorption of salts, regulation of cell volume [6], regulation of cell cycle [7] and production of reactive oxygen species (ROS) that act as second messengers [8]. CLIC1 has also been shown to play a role in maintaining structure of intracellular organelles by interaction between membrane and cytoskeleton [5].

Being the most intensely studied out of a recently described family of 7 proteins [9], the therapeutic importance of CLIC1 is becoming increasingly known. Recent studies have linked CLIC1 overexpression to cancerous tumors and its production may correlate with neurodegenerative disorders, leading to research into the potential use of CLIC1 as a biomarker or suitable therapeutic target for inflammatory disease therapy. The following critical review will investigate the structure of CLIC1, its regulation, and its role in chloride channel activity in normal cells, as well as explore the possibilities of CLIC1 use as therapeutic markers for cancer and neurodegenerative diseases.

\section{Structure and Metamorphosis of CLIC1}

CLIC1 is a 241 amino acid ion channel protein with two discrete $\mathrm{N}$ and C-domains linked by a proline-rich loop [5]. Structurally, CLICs are homologous to glutathione S-transferases (GST), a class of metabolic isozymes that conjugates the reduced form of glutathione (GSH) to electrophilic xenobiotics prior to detoxification and elimination [10]. The N-domain of CLIC1 (residues 1-90) has a thioredoxin fold consisting of a four-stranded mixed $\beta$-sheet plus three $\alpha$-helices while the C-domain is all helical (Figure 1) and closely resembles the $\Omega$ class of the glutathione- s-transferase (GST) with two exceptions: the position of the carboxyl-terminal helix h9, and the insertion of a highly negatively charged loop [5]. This loop, extending between helices h5 and h6 (Pro 147 - Gln 164), is a distinctive feature of CLIC1 and may be important in protein-protein interactions [4] (Figure 1).

CLIC1 falls into the rare category of 'metamorphic proteins' [1], as it can exist in two forms: as an integral membrane protein in intracellular compartments e.g., nuclear membrane or endoplasmic reticulum with ion channel function or as a soluble globular protein in the cytoplasm, with well-formed GSH binding site that can form a covalent complex with GSH. Under oxidizing conditions and low $\mathrm{pH}$, the soluble protein can undergo a reversible structural modification to an integral

*Corresponding author: Simon J. Myers, Neuro-Cell Biology Laboratory, Western Sydney University, Australia, Tel: +61-2-46203383; Fax: +61-2-46203025; E-mail: s.myers@uws.edu.au

Received: February 06, 2016; Accepted: February 15, 2016; Published February 18,2016

Citation: Nguyen H, Lauto A, Shanu A, Myers SJ (2016) Chloride Intracellular Channel Protein 1 and its Role in Neurodegenerative Disorders and Cancerous Tumors. Biochem Anal Biochem 5: 249. doi:10.4172/2161-1009.1000249

Copyright: $\odot 2016$ Nguyen $\mathrm{H}$, et al. This is an open-access article distributed under the terms of the Creative Commons Attribution License, which permits unrestricted use, distribution, and reproduction in any medium, provided the original author and source are credited. 
membrane form, resulting in the four-stranded mixed $\beta$-sheet being transformed into helices and loops [4] (Figure 2). When transitioning to this oxidized state, the crystal structure of CLIC1 is drastically changed as a large rearrangement of the $\mathrm{N}$-terminal domain occurs [5], allowing the formation of a non-covalent dimer consisting of only helices [4]. The oxidized CLIC1 dimer, stabilized by an intramolecular disulfide bond, maintains the ability to form chloride channels in artificial bilayers and vesicles, whereas a reducing environment will inhibit the formation of these ion channels [3,4] (Figure 2).

As well as sharing a weak sequence homology with the GST superfamily, particularly the $\Omega$ class of GST (Figure 3 ), the soluble monomeric form of CLIC1 also has a redox-active site resembling glutaredoxin on the $\mathrm{N}$-domain [1,5]. Glutaredoxins are a group of redox enzymes with GSH binding site, that help maintain cellular redox balance by oxidising GSH to form glutathione disulfide (GSSG), which in turn is reduced by glutathione reductase (GR) enzyme [11]. The redox-active site in CLIC1, like glutaredoxin, has a redox-reactive
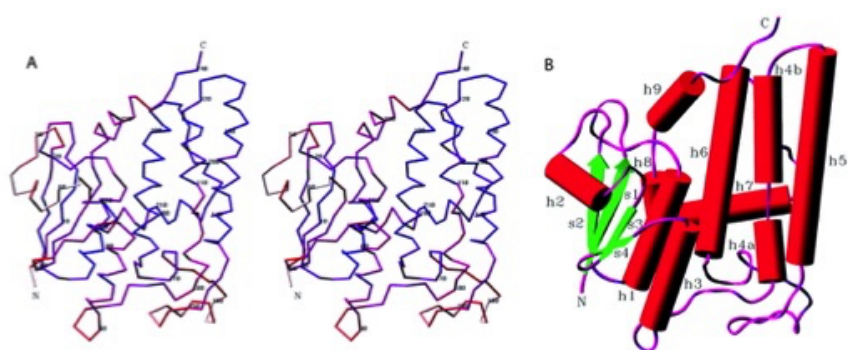

Figure 1: Structure of CLIC1 protein. A, a stereo diagram showing a CLIC1 monomer. The $\mathrm{N}$-domain is on the left, $\mathrm{C}$ - domain on the right with the GSH site at the top of the molecule in the inter-domain cleft. The backbone has been coloured such that blue denotes low and red denotes high. B, a schematic showing arrangement of secondary structural elements in CLIC1, with similar view to $\mathrm{A}[5]$.

A
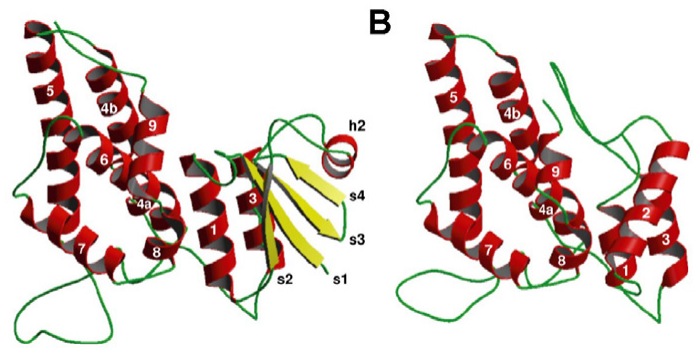

Figure 2: CLIC1 structural transition. Structural transition in CLIC1. A, a soluble monomer CLIC1 structure compared to $B$, the half-dimer structure observed after oxidation [41]
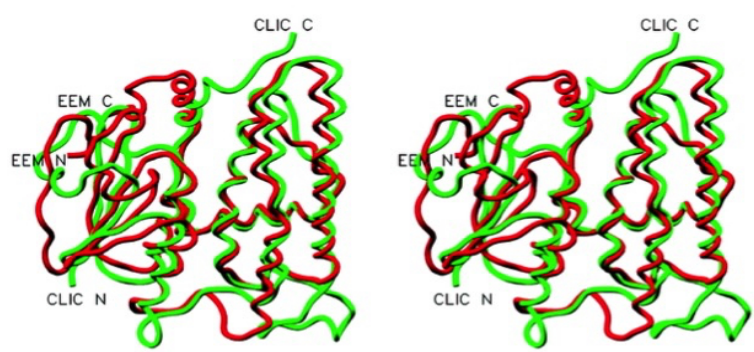

Figure 3: Structure comparison of CLIC1 and $\Omega$ GST. A comparison of the structure of CLIC1 with that of $\Omega$ GST. The figure shows a stereogram of the superposition of the CLIC1 structure (green) with that of the $\Omega$ class GST (red) [5]

\begin{tabular}{|l|c|}
\hline Cl efflux post CLIC1 inhibitor treatment & CI Efflux \%/s \\
\hline Group & $0.83 \pm 0.05(n=3)$ \\
\hline Control Vesicles & $1.92 \pm 0.36(n=2)$ \\
\hline CLIC1 Vesicles & $0.48 \pm 0.05(n=2)^{\star}$ \\
\hline CLIC1-IAA Vesicles &
\end{tabular}

Table 1: Inhibition of CLIC1 activity caused by the presence of IAA. Efflux rates were determined from vesicles mixed with control buffer (Control), $20 \mu \mathrm{g}$ of CLIC1 (CLIC), $20 \mu \mathrm{g}$ of CLIC1 in the presence of $50 \mu \mathrm{M}$ indanyloxyacetic acid-94 (IAA). * significantly lower to $\mathrm{Cl}$ efflux of CLIC1 vesicles $(P<0.02)[14]$.

\begin{tabular}{|l|c|}
\hline Cl efflux in heat-denatured CLIC1 proteins & CI Efflux \%/s \\
\hline Group & $0.40 \pm 0.06(n=2)$ \\
\hline Control Vesicles & $1.63 \pm 0.07(n=2)$ \\
\hline CLIC1 Vesicles & $0.652 \pm 0.004(n=2)^{\star}$ \\
\hline CLIC1- heat denatured
\end{tabular}

Table 2: Inhibition of CLIC1 activity through denaturation. Valinomycin-dependent efflux rates were determined in the absence of added protein (Control), in the presence of $4 \mu \mathrm{g}$ of CLIC1 (CLIC), and $4 \mu \mathrm{g}$ of CLIC1 that has been heated to $95^{\circ} \mathrm{C}$ for 5 min before the assay (Boiled). ${ }^{*}$ significantly lower to $\mathrm{Cl}$ efflux of CLIC1 vesicles $(P<0.001)[14]$

cysteine (Cys 24) that is capable of forming a covalent-mixed intramolecular disulfide bond (Cys24-Cys59) with GSH [12]. Mutational studies show that both Cys24 and Cys59 are required for channel activity $[4,13]$ (Figure 3 ).

\section{Regulation of CLIC1}

\section{Protein concentration}

CLIC1 membrane conductance can be regulated by its concentration. Studies carried out over a range of CLIC1 concentrations and corresponding rates of valinomycin-dependent chloride efflux showed that increasing amounts of CLIC1 protein leads to increasing chloride efflux rate [14]. At low protein concentrations, an essentially linear relationship between protein concentration and channel activity exists, whereas at a higher concentration, the rate of chloride efflux increases and then become saturated, resulting in a decreasing rate of increase of channel activity. This increase in channel activity can be partially attributed to the variations in protein-to-lipid ratios, where a high protein concentration, and consequently high protein-to-lipid ratio, will increase the possibility of multiple insertion events in a single vesicle [14]. The resultant increase in vesicles with multiple channels provides explanation to the increase of chloride channel activity.

\section{Presence of inhibitors}

The presence of inhibitors can greatly reduce CLIC1 function. IAA, an indanyloxyacetic acid derivative which is an inhibitor of microsomal chloride channel activity, has been found to significantly inhibit CLIC1 mediated valinomycin-dependent chloride efflux [14]. IAA, particularly IAA94 is a small and highly hydrophobic molecule that binds to the ion channel pore of CLIC1 with high affinity [9]. In the presence of IAA, the chloride efflux rate comparatively decreases, where a clear difference in chloride efflux rate between CLIC1 with and without the presence of the inhibitor is identifiable [14] (Tables 1 and 2).

\section{Hydrogen ion concentration}

CLIC1 channel activity is also strongly dependent on $\mathrm{pH}$. Measurement of the association of CLIC1 with liposomes at pH 6.5 and $\mathrm{pH} 7.4$ indicates that the interaction of CLIC1 with lipid bilayers is favored by lower $\mathrm{pH}$ levels [1]. Studies suggests the existence of a threshold between $\mathrm{pH} 6$ and 6.5, with the time needed to observe a functional ion channel tripling as the $\mathrm{pH}$ increased from range 5-6 to 


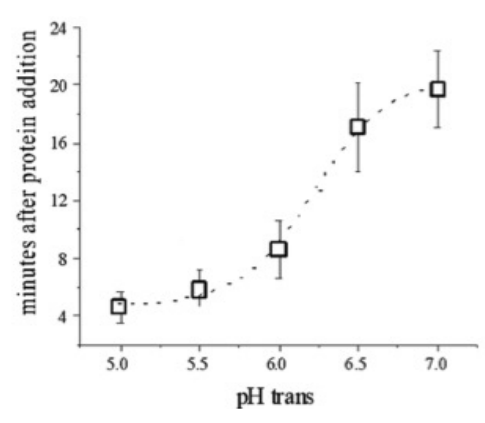

Figure 4: Delay in CLIC1 membrane insertion relative to $\mathrm{pH}$. CLIC1 single-channe activity versus $\mathrm{pH}$. The time of the first functional insertion in the lipid bilayer significantly increases as the $\mathrm{pH}$ is increased from range 5-5.5 to 6.5-7 [1].

range 6.5-7 (Figure 4) [1]. An explanation for these results lies in the relationship between a decrease in $\mathrm{pH}$ and in the increased probability of CLIC1 protein membrane insertion [14]. As the $\mathrm{pH}$ is lowered, it approaches the $\mathrm{pI}$ of the protein ( $\mathrm{pI} 4.5$ ). As a result, electrostatic repulsion effects between channel subunits are lowered, and the formation of an active ion channel is favored. Additionally, the decrease in $\mathrm{pH}$ can also decrease the activation barrier that must be overcome for CLIC1 to transition from the soluble form to the membrane conformation, allowing CLIC1 to more easily integrate into the lipid bilayer [1] (Figure 4).

\section{Redox regulation of CLIC1 membrane insertion}

Presence of GSH binding site in CLIC1 suggests that chloride ion channel activity, promoted by the transition from a reduced, monomeric, cytoplasm-soluble state to an oxidized soluble non-covalent dimeric state, may be regulated via a GSH-dependent redox mechanism in vivo [5]. It is possible that CLIC1 uses the GSH site for targeting the chloride channel to a particular subcellular location. However, in the cytoplasm, GSH is present and the conditions are strongly reducing [5]. Thus, the soluble form of CLIC1 is likely to be free of glutathione attachment and Cys $\wedge 24$ is likely to be in a reduced state, preventing the formation of ion channels. The presence of oxygen species such as hydrogen peroxide alters this resting state of soluble CLIC1, possibly enabling channel activity [5]. Although it has been independently proved that oxidation promotes dimer formation and membrane insertion, it is still not clear whether dimerization is significant for membrane insertion. In fact, both monomer and dimer forms of CLIC1 has been found to be able to undergo ion channel formation in artificial membranes [2]. Albeit the Cys24-Cys59 disulfide bond stabilizing the dimer form, which is required for ion channel conductance, it has been proved that the existence of Cys24-Cys59 bond effectively restrains the protein from membrane integration [15]. Thus the model Littler suggests for CLIC1 membrane insertions seems plausible whereby in solution, under oxidizing conditions, CLIC1 monomeric form undergoes structural transition to form an unstable Cys24-Cys59 disulfide bond, exposing a hydrophobic domain which in absence of lipid layer, forms a conformationally stable dimer masking the hydrophobic domain. However, in presence of the lipid layer, it docks itself to the membrane using the hydrophobic domain; the actual fate of CLIC1 depending on the concentration of the lipid layer [2].

\section{CLIC1 insertion into lipid membrane}

The traditional route of integral membrane synthesis and processing involves the protein undergoing integral membrane synthesis by ribosomes bound to the endoplasmic reticulum (ER) and remaining lodged in the ER membrane until its eventual delivery by the Golgi complex to its final membrane destination $[1,16]$. However, CLIC1 challenges this traditional assumption regarding protein insertion into membranes, being capable of spontaneous insertion into lipid bilayers directly from their soluble form [16]. Additionally, isolated CLIC1 can form functional ion channels in an artificial lipid bilayer with identical electrophysiological characteristics with that of cell membranes [1]

CLIC1 membrane insertion occurs in a multi-step process. Firstly, under oxidizing conditions, the globular soluble form of the protein unfolds, exposing a hydrophobic face. Next, cholesterol will act as a potential binding site on the membrane, to which CLIC1 can attach, and subsequently insert into the membrane [16]. Once associated with the membrane, it has also been suggested that cholesterol aids with the higher order quaternary structural arrangement of the protein into its final conductive state in the ion channel with the amino terminus on the exterior, and an inward cytoplasmic C-terminus [9].

\section{CLIC1 as an oxidoreductase}

A recent study on CLIC1 suggests it also exhibits oxidoreductase activity in addition to its role as a chloride ion channel [17]. In this study, CLIC1 protein expressed in E. coli was extracted and isolated using chromatography and used in its reduced monomeric and oxidised dimeric forms to detect CLIC1 activity under various conditions. It was demonstrated that CLIC1 in its monomeric and dimeric forms, reduced 2-hydroxyethyl disulphide (HEDS), a glutaredoxin substrate, in presence of GSH and GR as demonstrated by the oxidation of $\mathrm{NADPH}$, indicating its oxidoreductase activity. However, it has been proposed only the monomeric form possess the antioxidant property, and the dimer is reduced to monomeric form in presence of GSH [17]. Mutational studies using C24A, C24S and C59A revealed that the Cys24 moiety was essential for the oxidoreductase activity but not the Cys59 [17]. Further studies also revealed that the oxidoreductase activity was inhibited by presence of ion channel blocker IAA but not cholesterol indicating that although both functions might be distinct to each other, enzymatic properties of CLIC1 may direct the ion channel function [17]. Establishment of CLIC1 as an oxidoreductase exposes it as a moonlighting protein that has multiple functions that maximise efficiency of the proteome [18]. This also shows that presence of oxidants is not the only factor that designates the fate of CLIC1, when CLIC1 itself can act as an antioxidant. As described earlier, multiple other factors such as presence of cholesterol [16], $\mathrm{pH}$ [1] and membrane phospholipid concentration [14] also directs the localisation and function of CLIC1.

\section{The role of the CLIC1 protein in non-disease states}

Under oxidizing conditions and low $\mathrm{pH}$, CLIC1, can integrate into synthetic lipid bilayers and vesicles to function as chloride ion channels [3,4]. Like other classes of chloride channels such as the ligand-gated receptor channels and CIC chloride ion channels, this renders the CLIC1 protein capable of regulating a number of crucial cellular processes including cell volume, stabilization of cell membrane potential, trans-epithelial transport, and maintenance of intracellular $\mathrm{pH}[3,5]$.

CLIC1 ion channels were shown to be reversibly inhibited by cytosolic F-actin in the absence of other proteins [3]. A reversal of this effect can be achieved using cytochalasin, which disrupts F-actin. This finding presents a new possibility for CLIC1, being an indication that CLIC1, like other actinregulated membrane CLICs, can possess non-channel functions such as cell cycle regulation, cell differentiation and cell apoptosis [19]. Like actinregulated membrane CLICs, CLIC1 can modify solute transport during various stages in the cell cycle and cell migration [3]. CLIC1 is also involved 
in apical membrane recycling in several columnar epithelia tissue samples including the small intestine and renal proximal tubes [19]. CLIC1 is also detected in the epithelia of airways, the gall bladder, pancreas, stomach, small intestine and colon [20].

Regulation of the cell cycle is another role that can also be attributed to CLIC1. This is supported by study on Chinese hamster ovary cells, which showed that the use of CLIC1 blockers can inhibit cells in the G2/M stage of the cell cycle [7]. In this stage, the ion channel is selectively expressed on the plasma membrane, and the absence of CLIC1 will prevent CLIC1-mediated changes in cell volume and hinder the completion of mitosis so the division of the cell is prevented [3,7].

CLIC1 is also found to be highly expressed in macrophages which are key cells in adaptive immunity [21]. These macrophages have a crucial role in tissue homeostasis, wound repair and host defense. In resting macrophages, CLIC1 is widely distributed in the cytoplasm [9]. However, in activated macrophages, CLIC1 moves to the phagosomal membrane and plays a role in regulating phagosomal acidification, and consequently, control the regulation of other phagosomal functions such as proteolysis through its ion channel activity [9]. In the absence of CLIC1, an elevated phagosomal $\mathrm{pH}$ results in a decrease of phagosomal proteolytic activity and reduced ROS production [9]. When tested in vitro with mice, it was demonstrated that mice with CLIC1-negative macrophages are protected from developing chronic joint inflammation, which provides evidence for the use of CLIC1 as useful target for the development of anti-inflammatory drugs $[9,21]$.

\section{The role of CLIC1 protein in diseased state}

CLIC1 appears to have a role in pathologies characterized by oxidative stress. Recent studies suggest that redox sensitive CLIC1 also extend its effector mechanisms using ROS as second messengers. Silencing of CLIC1 mRNA was found to decrease phosphorylation of extracellular signal related kinase $1 / 2$ (p-ERK1/2) and matrix metalloproteinase 2, 9 (MMP 2, 9) [22], which are both downstream effectors of oxidative stress pathways, a key feature of cancer pathology $[23,24]$. Furthermore, studies show localization of CLIC1 on plasma membrane during G2/ M phase of cell cycle, during which the current density is twice that of the resting phase (G1/ S) of the cell cycle [7], suggesting its possible role in tumor pathology. CLIC1 overexpression has also been found to be required for amyloid-beta induction of ROS by activated microglia, which is a precursor of neurodegenerative disorders such as Alzheimer disease [25].

\section{Expression of CLIC1 in cancerous tumors}

CLIC1 is overexpressed in many cancerous tumors, including lymph node metastasis and lymphatic invasion [26]. This is because neoplastic transformation, particularly active migration and high rates of proliferation into non-neoplastic tissues, requires partial or total participation of the chloride channel [27]. Thus, during tumorigenesis, the increased permeability of chloride and potassium selective pores allows CLIC1 to become the most active channel, accounting for its overexpression in cancerous tumors. This is confirmed in human primary hepatic tumors where studies showed a higher presence of CLIC1 protein in cancerous tissues compared to corresponding noncancerous tissue [28]. As CLIC1 is overexpressed in cancerous tissues, staining cells with anti-CLIC1 antibody can offer detection of tumorous cells, suggesting that CLIC1 can potentially become an effective prognostic marker for these cancers, and also a means for drug targeting [29].

Furthermore, overexpression of CLIC1 can also enhance migration and invasion of carcinogenic cells. In gall bladder carcinoma cells, CLIC1 promotes cell motility and invasion of the carcinogenic cells, whereas interference of CLIC1 RNA can significantly decrease cell motility and the invasive potency of the cells in vitro [30]. The same explanation could be used to interpret the role of CLIC1 in gastric cancer cells, where the 5-year survival rate of the low CLIC1 expression group to be 1.72 times higher than that of the high expression group [29]. Additionally, it was also noted that by simply reducing CLIC1 expression through the use of a specific CLIC1 blocker, its function can be suppressed, resulting in a reduction of the migration ability of endothelial cells [3,31-35].

\section{CLIC1 and neurodegeneration}

CLIC1 is overexpressed in Alzheimer's disease, a currently untreatable neurodegenerative disease characterized by the presence of amyloid plaques formed by abundant extracellular deposits of $A \beta$ [36]. At a cellular level, the deposits of amyloid- $\beta$ activate microglia, which secretes potentially neurotoxic molecules as well as tumor necrosis factor- $\alpha$. (TNF- $\alpha$ ), a pro-inflammatory cytokine that also contributes to microglial neurotoxicity [37], leading to accelerated neuronal death (Figure 5). Microglial activation has been identified as a characteristic of a number of neurodegenerative diseases including multiple sclerosis, Parkinson disease, dementia, stroke, amyotrophic lateral sclerosis (ALS) and Creutzfeldt-Jacob disease [38]. ROS are thought to comprise a vast majority of neurotoxic molecules generated by activated microglia. Activation of nicotinamide adenine dinucleotide phosphate oxidase (NADPH oxidase) constitutes a major source of ROS, which has been identified to play a crucial role in pathogenesis of ALS [39] Figure 5.

The activation of microglia also increases CLIC1 protein expression within 24 hours of incubation with $A \beta$ [21]. The overall increase in CLIC1 protein expression consequently directs the protein to the plasma membrane to perform its role as a chloride ion channel [31]. This accounts for substantial increase in plasma membrane CLIC1related ion channel activity accompanying cell activation, which is confirmed to be specific to the $A \beta$ peptide [21]

In light of the relationship between $A \beta$ microglial activation and CLIC1 expression, studies show that CLIC1 could be of 'therapeutic efficacy' in Alzheimer's disease [31]. A blockade of CLIC1 by the

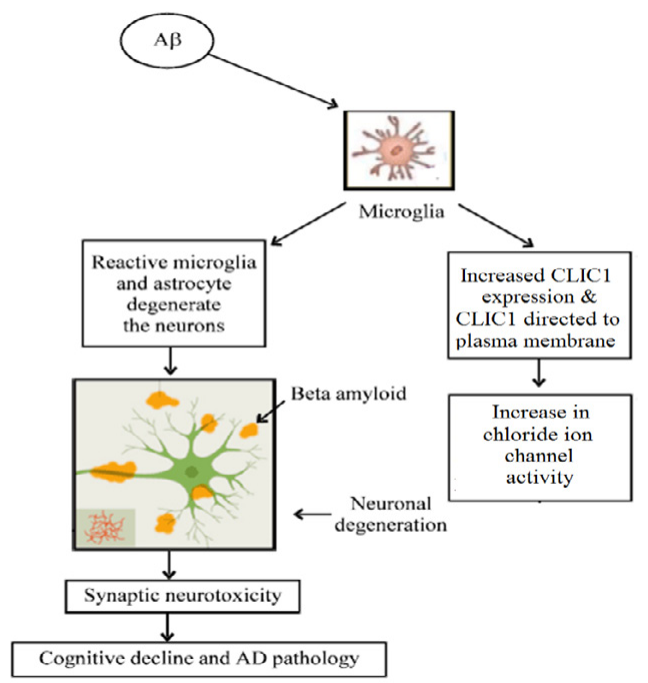

Figure 5: Microglial pathway leading to neuronal degeneration. Flow diagram outlining the process of neurodegeneration in Alzheimer's disease and its effect on CLIC1 expression [40]. 
CLIC1 ion channel blocker IAA-94 reduces the function of CLIC1 and stimulates phagocytosis of $A \beta$ in mononuclear phagocytes, as well as function as a neuroprotective agent in neuronal-microglial co-cultures [31]. This limits microglial activation, preventing further release of neurotoxic substances [21]. Additionally, CLIC channel blockers and small interfering RNA (siRNAs) also have the power to cease CLIC1 expression, completely inhibiting the release of TNF- $\alpha$ from microglial cells, and further reducing neuronal damage [21]. Therefore, with more research into methods to trigger a blockade of CLIC1, these results show the potential for CLIC1 to become a therapeutic target for Alzheimer's disease. Recently, our group has identified increased CLIC1 expression in patient lymphocytes collected from hereditary sensory neuropathy-type1 (HSN1) patients. HSN1 is a dominantly inherited peripheral sensory neuropathy characterised by sensory loss in the feet followed by distal muscle wasting and weakness [32]. It is clinically an axonal neuropathy affecting distal long nerves, which slowly spreads proximally over a lifetime. Although classified as a neurodegenerative disease, the pathological course of CLIC1 mediated neuronal degeneration followed by HSN1 would be expected to be different from the pathologies caused by microglial activation for obvious reasons. Increased accumulation of lipid droplets has been identified as a characteristic of HSN1 lymphoblasts [33] and also neurodegenerative disorders such as Alzheimer's and Parkinson's diseases [34,35], which is also indicative of anomalous lipid metabolism. It would useful to analyse the presence or absence of CLIC1 in these lipid droplets to identify if it plays a role in the pathophysiology of these diseases. Whether CLIC1 overexpression is a critical mediator of the pathology or just a secondary effector pathway that aggravates the condition also needs to be investigated.

\section{Conclusion}

Despite being a recently-cloned protein, research into the structure and functions of CLIC1 has revealed characteristics, namely its metamorphic nature, presence of a single transmembrane region, and its direct insertion into lipid membranes, that greatly distinguish it from other ion channel proteins [1]. Whilst there is still limited data on the biological role of CLICs, the chloride conductance of CLIC1 gives the protein a significant role in the regulation of crucial cellular processes and thus, provides an explanation for its high degree of conservation amongst a wide range of species [4].

As the role of CLIC1 in non-diseased states becomes clearer, the therapeutic importance of CLIC1 in diseased states is also becoming increasingly known, where the overexpression of CLIC1 is linked to many currently untreatable diseases including cancerous tumors [2629] and neurodegenerative disorders including Alzheimer's disease and ALS [21,31]. For this reason, further research into the role of CLIC1 and its regulation in cell membranes and organelles is critical, offering possibilities for its potential use as a suitable therapeutic target for neurodegenerative diseases [36-41].

\section{References}

1. Warton K, Tonini R, Fairlie WD, Matthews JM, Valenzuela SM, et al. (2002) Recombinant CLIC1 (NCC27) assembles in lipid bilayers via a $\mathrm{pH}$-dependent two state process to form chloride ion channels with identical characteristics to those observed in Chinese hamster ovary cells expressing CLIC1. The Journal of Biological Chemistry 227: 26003-26011.

2. Littler DR, Harrop SJ, Fairlie WD, Brown LJ, Pankhurst GJ, et al. (2004) The intracellular chloride ion channel protein CLIC1 undergoes a redox-controlled structural transition. J Biol Chem 279: 9298-9305.

3. Liao PC, Chang YH (2011) CLIC1 (chloride intracellular channel 1). Atlas of genetics and cytogenetics in oncology and haematology 16: 266-269.
4. Littler DR, Harrop SJ, Goodchild SC, Phang JM, Mynott AV, et al. (2010) The enigma of the CLIC proteins: ion channels, redox proteins, enzymes, scaffolding proteins? FEBS Letters 584: 1-10.

5. Harrop SJ, DeMaere MZ, Fairlie WD, Reztsova T, Valenzuela SM, et al. (2001) Crystal structure of the intracellular chloride ion channel CLIC1 (NCC27) at 1.4A Resolution. The Journal of Biological Chemistry 276: 44993-45000.

6. Tonini R, Ferroni A, Valenzuela SM, Warton K, Campbell TJ, et al. (2000) Functional characterization of the NCC27 nuclear protein in stable transfected CHO-K1 cells. FASEB J 14: 1171-1178.

7. Valenzuela SM, Mazzanti M, Tonini R, Qiu MR, Warton K, et al. (2000) The nuclear chloride ion channel NCC27 is involved in regulation of the cell cycle. J Physiol 529: 541-552.

8. Averaimo S, Milton RH, Duchen MR, Mazzanti M (2010) Chloride intracellula channel 1 (CLIC1): Sensor and effector during oxidative stress. FEBS Lett 584: 2076-2084.

9. Jiang L, Salao K, Li H, Rybicka JM, Yates RM, et al. (2012) Intracellular chloride channel protein CLIC1 regulates macrophage function through modulation of phagosomal acidification. Journal of Cell Science 124: 5479-5488.

10. Hayes JD, Flanagan JU, Jowsey IR (2005) Glutathione transferases. Annu Rev Pharmacol Toxicol 45: 51-88.

11. Grant CM (2001) Role of the glutathione/glutaredoxin and thioredoxin systems in yeast growth and response to stress conditions. Mol Microbiol 39: 533-541.

12. Singh H, Ashley R (2006) Redox regulation of CLIC1 by cysteine residues associated with the putative channel pore. Biophysical Journal 90: 1628-1638.

13. Valenzuela SM, Berkhan M, Porkovich A, Huynh T, Goyette J, et al. (2011) Soluble Structure of CLIC and S100 Proteins investigated by Atomic Force Microscopy. Journal of Biomaterials and Nanotechnology 2: 8-17.

14. Tulk BM, Kapadia S, Edwards JC (2002) CLIC1 inserts from the aqueous phase into phospholipid membranes, where it functions as an anion channel. American Journal of Physiology 282: 1103-1112.

15. Goodchild SC, Howell MW, Cordina NM, Littler DR, Breit SN, et al. (2009) Oxidation promotes insertion of the CLIC1 chloride intracellular channel into the membrane. Eur Biophys J 1: 129-138.

16. Valenzuela SM, Al Khamici H, Brown LJ, Almond OC, Goodchild SC, et al. (2013) Regulation of the membrane insertion and conductance activity of the metamorphic chloride intracellular channel protein CLIC1 by cholesterol. PLoS One 8: 1-8.

17. Khamici AH, Brown LJ, Hossain KR, Hudson AL, Ng JP, et al. (2015) Members of the chloride intracellular ion channel protein family demonstrate glutaredoxinlike enzymatic activity. PLoS One 10: e115699.

18. Jeffery CJ (1999) Moonlighting proteins. Trends in biochemical sciences 24 8-11.

19. Tung JT, Kitajewski J (2010) Chloride intracellular channel 1 functions in endothelial cell growth and migration. Journal of Angiogenesis Research 2 : $1-13$.

20. Ulmasov B, Bruno J, Woost PG, Edwards JC (2007) Tissue and subcellular distribution of CLIC1. BioMed Central Cell Biology 8: 1-18.

21. Novarino G, Fabrisi C, Tonini R, Denti MA, Malchiodi-Albedi F, et al. (2004) Involvement of the intracellular ion channel CLIC1 in microglia-mediated ß-amyloid-induced neurotoxicity. J Neurosci 24: 5322-5330.

22. Tian Y, Guan Y, Jia Y, Meng Q, Yang J (2014) Chloride intracellular channel 1 regulates prostate cancer cell proliferation and migration through the MAPK/ ERK pathway. Cancer Biother Radiopharm 29: 339-344.

23. Gan X, Wu L, Huang S, Zhong C, Shi H, et al. (2014) Oxidative stress-mediated activation of extracellular signal-regulated kinase contributes to mild cognitive impairment-related mitochondrial dysfunction. Free Radic Biol Med 75: 230240.

24. Lehner C, Gehwolf R, Tempfer H, Krizbai I, Hennig B, et al. (2011) Oxidative stress and blood-brain barrier dysfunction under particular consideration of matrix metalloproteinases. Antioxid Redox Signal 15: 1305-1323.

25. Milton RH, Abeti R, Averaimo S, DeBiasi S, Vitellaro L, et al. (2008) CLIC1 function is required for beta-amyloid-induced generation of reactive oxygen species by microglia. J Neurosci 28: 11488-11499. 
Citation: Nguyen H, Lauto A, Shanu A, Myers SJ (2016) Chloride Intracellular Channel Protein 1 and its Role in Neurodegenerative Disorders and Cancerous Tumors. Biochem Anal Biochem 5: 249. doi:10.4172/2161-1009.1000249

26. Chen $C D$, Wang CS, Huang YH, Chien KY, Liang Y, et al. (2007) Overexpression of CLIC1 in human gastric carcinoma and its clinicopathological significance. Proteomics 7: 155-167.

27. Peretti M, Angelini M, Savalli N, Florio T, Yuspa SH, et al. (2014) Chloride channels in cancer: focus on chloride intracellular 1 and 4 (CLIC1 and CLIC4) proteins in tumor development and as novel therapeutic targets. Biochimica et Biophysica Acta 1848: 2523-2531.

28. Zhang S, Wang X, Yin ZY, Zhao WX, Zhou JY, et al. (2013) Chloride intracellular channel 1 is overexpression in hepatic tumor and correlates with a poor prognosis Acta Pathologica, Microbiologica Et Immunologica Scadinavica 121: 1047-1053.

29. Ma PF, Chen JQ, Wang Z, Liu JL, Li BP (2012) Function of chloride intracellular channel 1 in gastric cancer cells. World Journal of Gastroenterology 18: 3070 3080 .

30. Wang P, Zeng Y, Liu T, Zhang C, Yu PW, et al. (2014) Chloride intracellular channel 1 regulates colon cancer cell migration and invasion through ROS/ ERK pathway. World J Gastroenterol 20: 2071-2078.

31. Paradisi S, Matteucci A, Fabrisia C, Denti MA, Abeti R, et al. (2008) Blockade of chloride intracellular ion channel 1 stimulates A-beta phagocytosis. J Neurosci Res 86: 2488-2498.

32. Dawkins JL, Brahmbhatt S, Auer-Grumbach M, Wagner K, Hartung HP, et al. (2002) Exclusion of serine palmitoyltransferase long chain base subunit 2 (SPTLC2) as a common cause for hereditary sensory neuropathy. Neuromuscul Disord 12: 656-658.
33. Marshall LL, Stimpson SE, Hyland R, Coorssen JR, Myers SJ (2014) Increased lipid droplet accumulation associated with a peripheral sensory neuropathy. $J$ ChemBiol 7: 67-76.

34. Lane RM, Farlow MR (2005) Lipid homeostasis and apolipoprotein E in the development and progression of Alzheimer's disease. J Lipid Res 46: 949-968.

35. Cole NB, Murphy DD, Grider T, Rueter S, Brasaemle D, et al. (2002) Lipid droplet binding and oligomerization properties of the Parkinson's disease protein alpha-synuclein. J Biol Chem 277: 6344-6352.

36. Glenner GG, Wong CW (1984) Alzheimer's disease: initial report of the purification and characterization of a novel cerebrovascular amyloid protein Biochemical and biophysical research communications 120: 885-890.

37. Tobinick E, Gross H, Weinberger A, Cohen H (2006) TNF-alpha modulation fo treatment of Alzheimer's disease: A 6-month pilot study. Med Gen Med 8: 25

38. Skaper SD, Facci L, Giusti P (2013) Intracellular ion channel CLIC1: involvement in microglia-mediated $B$-amyloid peptide(1-42) neurotoxicity. Neurochem Res 38: 1801-1808.

39. Boillee S, Cleveland DW (2008) Revisiting oxidative damage in ALS: microglia Nox, and mutant SOD1. J Clin Invest 118: 474-478.

40. Rai S, Kamat PK, Nath C, Shukla R (2014) Glial activation and synaptic neurotoxicity in Alzheimer's disease: a focus on neuroinflammation. Pharmacologia 5: 286-297.

41. UNSW Protein Structure Laboratory. Structural transition in CLIC1. 